\title{
CHARITÉ
}

UNIVERSITÄTSMEDIZIN BERLIN

\section{Interaction of HPA axis genetics and early life stress shapes emotion recognition in healthy adults}

Corinna Hartling, Yan Fan, Anne Weigand, Irene Trilla, Matti Gärtner, Malek Bajbouj, Isabel Dziobek, Simone Grimm

\section{Document type}

Postprint (accepted version)

This version is available at

https://doi.org/10.17169/refubium-26260

\section{Citation details}

Hartling C, Fan Y, Weigand A, Trilla I, Gärtner M, Bajbouj M, et al. Interaction of HPA axis genetics and early life stress shapes emotion recognition in healthy adults. Psychoneuroendocrinology.

[Online] Elsevier BV; 2019;99: 28-37. DOI: 10.1016/j.psyneuen.2018.08.030

\section{Terms of use}

This work is licensed under a Creative Commons Attribution-NonCommercial-NoDerivatives 4.0 International license: https://creativecommons.org/licenses/by-nc-nd/4.0/ 


\section{Accepted Manuscript}

Title: Interaction of HPA axis genetics and early life stress shapes emotion recognition in healthy adults

Authors: Corinna Hartling, Yan Fan, Anne Weigand, Irene Trilla, Matti Gärtner, Malek Bajbouj, Isabel Dziobek, Simone Grimm

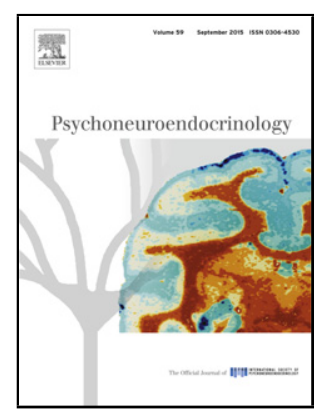

PII: S0306-4530(17)31563-9

DOI: https://doi.org/10.1016/j.psyneuen.2018.08.030

Reference: PNEC 4042

To appear in:

Received date: $\quad$ 25-12-2017

Revised date: $\quad 15-8-2018$

Accepted date: $\quad 22-8-2018$

Please cite this article as: Hartling C, Fan Y, Weigand A, Trilla I, Gärtner M, Bajbouj M, Dziobek I, Grimm S, Interaction of HPA axis genetics and early life stress shapes emotion recognition in healthy adults, Psychoneuroendocrinology (2018), https://doi.org/10.1016/j.psyneuen.2018.08.030

This is a PDF file of an unedited manuscript that has been accepted for publication. As a service to our customers we are providing this early version of the manuscript. The manuscript will undergo copyediting, typesetting, and review of the resulting proof before it is published in its final form. Please note that during the production process errors may be discovered which could affect the content, and all legal disclaimers that apply to the journal pertain. 
Hartling et al: Interaction of HPA axis genetics and early life stress shapes emotion recognition in healthy adults

Correspondence should be addressed to:

Corinna Hartling, M.Sc. psychology

Klinik und Hochschulambulanz für Psychiatrie und Psychotherapie

Charité - Universitätsmedizin Berlin, corporate member of Freie Universität Berlin,

Humboldt-Universität zu Berlin, and Berlin Institute of Health

Campus Benjamin Franklin (CBF)

Hindenburgdamm 30

12203 Berlin

corinnahartling@gmail.com

Financial support: The study was supported by the Deutsche Forschungsgemeinschaft (DFG) [DFG-Grant GR 4510/2-1 to Simone Grimm]

Conflict of interest: none

For all figures please use colour only online. 
Hartling et al: Interaction of HPA axis genetics and early life stress shapes emotion recognition in healthy adults

\title{
Interaction of HPA axis genetics and early life stress shapes emotion recognition in healthy adults
}

Authors: Corinna Hartling ${ }^{\mathrm{a}}$, Yan Fan ${ }^{\mathrm{a}}$, Anne Weigand ${ }^{\mathrm{a}, \mathrm{b}}$, Irene Trilla ${ }^{\mathrm{b}}$, Matti Gärtner ${ }^{\mathrm{a}}$, Malek Bajbouj ${ }^{\mathrm{a}}$, Isabel Dziobek $^{\mathrm{b}}$, Simone Grimm ${ }^{\mathrm{a}, \mathrm{c}, \mathrm{d}}$

a Klinik und Hochschulambulanz für Psychiatrie und Psychotherapie, Charité - Universitätsmedizin Berlin, corporate member of Freie Universität Berlin, Humboldt-Universität zu Berlin, and Berlin Institute of Health, Campus Benjamin Franklin, Hindenburgdamm 30, 12203 Berlin, Germany

b School of Mind and Brain, Humboldt-Universität zu Berlin, Luisenstraße 56, 10117 Berlin, Germany

c Department of Psychiatry, Psychotherapy and Psychosomatics, Psychiatric Hospital, University of Zurich, 8032 Zurich, Switzerland

d MSB Medical School Berlin, Calandrellistr. 1-9, 12247 Berlin

Highlights

- HPA axis genetic profile predicts cortisol increase after psychosocial stress

- Early life stress effect on emotion recognition depends on HPA axis genetic profile

- HPA axis genetic profiles may inform our understanding of early life stress effects

\begin{abstract}
Background: Early life stress (ELS) affects facial emotion recognition (FER), as well as the underlying brain network. However, there is considerable inter-individual variability in these ELS-caused alterations. As the hypothalamic-pituitary-adrenal (HPA) axis is assumed to mediate neural and behavioural sequelae of ELS, the genetic disposition towards HPA axis reactivity might explain differential vulnerabilities.
\end{abstract}

Methods: An additive genetic profile score (GPS) of HPA axis reactivity was built from 6 SNPs in 3 HPA axisrelated genes (FKBP5, CRHR1, NR3C1). We studied two independent samples. As a proof of concept, GPS was tested as a predictor of cortisol increase to a psychosocial challenge (MIST) in a healthy community sample of $\mathrm{n}=40$. For the main study, a sample of $\mathrm{n}=170$ completed a video-based FER task and retrospectively reported ELS experiences in the Childhood Trauma Questionnaire (CTQ).

Results: GPS positively predicted cortisol increase in the stress challenge over and above covariates. CTQ and genetic profile scores interacted to predict facial emotion recognition, such that ELS had a detrimental effect on emotion processing only in individuals with higher GPS. Post-hoc moderation analyses revealed that, while a less stress-responsive genetic profile was protective against ELS effects, individuals carrying a moderate to high GPS were affected by ELS in their ability to infer emotion from facial expressions.

Discussion: These results suggest that a biologically informed genetic profile score can capture the genetic disposition to HPA axis reactivity and moderates the influence of early environmental factors on facial emotion recognition. Further research should investigate the neural mechanisms underlying this moderation. The GPS used here might prove a powerful tool for studying inter-individual differences in vulnerability to early life stress. Keywords: early life stress; HPA axis; G-X-E-interaction; emotion recognition; genetic profile 
Hartling et al: Interaction of HPA axis genetics and early life stress shapes emotion recognition in healthy adults

\section{Introduction}

For humans navigating a social world, facial expressions are a valuable source of information about other's emotional states and adequate behaviour in social interactions. Competency in inferring emotions from facial expressions (facial emotion recognition; FER) emerges already in infancy and continues to develop with increasing refinement until adolescence (Leppänen and Nelson, 2009). Early life stress (ELS; i.e. conditions during childhood that threaten the emotional or physical well-being and exceed the child's coping resources), however, affects this development (da Silva Ferreira et al., 2014). Children who underwent maltreatment show a generally poorer performance in FER tasks than their non-maltreated peers (Pollak et al., 2000), and adults with a history of ELS show similar impairments (Germine et al., 2015; Jedd et al., 2015), suggesting that ELS effects on emotion processing persist throughout the lifespan.

Such enduring alterations in emotion processing following ELS point to potential changes in emotion-related brain circuits. Facial emotion recognition involves a network of regions that critically comprises the amygdala (Fusar-Poli et al., 2009). Although neurogenesis in the amygdala is completed by birth, it undergoes substantial changes to its anatomy and connectivity with prefrontal regions postnatally (Leppänen and Nelson, 2009), making it susceptible to disruptive effects of early life stress. A growing body of literature indeed documents structural, functional and connectomic changes of the amygdala following ELS (Fareri and Tottenham, 2016; Teicher et al., 2016). Specifically, ELS has been associated with an increased excitability of the amygdala towards emotional faces (Dannlowski et al., 2012; McCrory et al., 2011; van Harmelen et al., 2013), as well as changed amygdala-prefrontal connectivity during emotion processing (Jedd et al., 2015).

Although these lines of evidence consistently demonstrate changes in emotion processing and the involved brain network following ELS, it is still not fully understood what causes this altered development. Moreover, there are considerable inter-individual differences in how severely people are affected by ELS, both regarding behaviour and neural endophenotypes (da Silva Ferreira et al., 2014; Teicher et al., 2016), 
Hartling et al: Interaction of HPA axis genetics and early life stress shapes emotion recognition in healthy adults

the origins of which remain unclear. Investigating this variability may help to identify the neurobiological mechanisms underlying the sequelae of early life stress and may inform preventive and therapeutic interventions.

The neuroendocrine stress response has been suggested as the main mediating factor in ELS-caused changes to the amygdala, as it is rich in glucocorticoid receptors (Lupien et al., 2009) and both endogenous cortisol and pharmacologic agonism of the HPA axis trigger amygdala activation (Bogdan et al., 2016). In animals, protracted GR stimulation in the amygdala alters synaptic functioning between the amygdala and frontal regions in a pattern similar to ELS-caused changes in humans (Myers et al., 2014). These data suggest that the HPA axis plays a role in how early life stress brings about changes to the core structure in facial emotion recognition. Accordingly, individual differences in HPA axis function might be moderating the effects environmental stress exerts on FER.

HPA axis activity is highly heritable (Federenko et al., 2004) and multiple genetic association studies have identified polymorphisms in genes coding for HPA axis-related proteins that are associated with variability in the cortisol response to a psychosocial or pharmacological challenge (Supplementary Table 3; for an overview see DeRijk, 2009). Single nucleotide polymorphisms (SNPs) in three such genes, CRHR1, NR3C1, and FKBP5, are studied here (the selection process is outlined below). The CRHR1 gene codes for a receptor that binds corticotropin-releasing hormone and is thus a mediator of HPA axis activation. By contrast, the $N R 3 C 1$ gene which codes for the glucocorticoid receptor and the $F K B P 5$ gene which codes for a binding protein fine-tuning GR availability, are involved in the negative feedback loop of the HPA axis (Pagliaccio et al., 2014).

Further, HPA axis relevant SNPs moderate the effects of early life stress exposure. In particular, neural ecophenotypes of early life stress (increased amygdala volume, increased reactivity to emotional stimuli, and decreased connectivity with prefrontal regions) are more pronounced in individuals with a genetic disposition towards greater HPA axis activity (Di Iorio et al., 2017; Holz et al., 2015; Pagliaccio et 
Hartling et al: Interaction of HPA axis genetics and early life stress shapes emotion recognition in healthy adults

al., 2015a, 2014; White et al., 2012). Similarly, SNPs conferring greater HPA axis activity might also moderate the impairments of emotion recognition following ELS. Specifically, SNPs coding for important switch points of the HPA axis could potentiate the neuroendocrine and subsequent downstream effects of exposure to environmental stress, but otherwise have little differential effect in the absence of stress.

Based on these previous findings, the present study examined whether a genetic profile score (GPS) reflecting functionally relevant variation in 6 loci in 3 genes coding for integral HPA axis proteins moderated the relationship between ELS and facial emotion recognition. This approach of building biologically informed genetic profile scores has previously been capable to significantly explain variance of endophenotypes when individual SNPs would not (Nikolova et al., 2011; Pagliaccio et al., 2014). This approach is especially valuable for G-x-E-interaction studies that are constrained by both the low frequency of risk-conferring variants and the frequency of environmental stressors. Genetic profile scores have the advantage of assessing higher-order function of a signalling cascade instead of the effects on single components of a signalling cascade that single SNPs might exert. The phenotype of HPA axis activity has previously been mapped in different polygenic score models (Bogdan et al., 2016) that we used to guide our approach (Pagliaccio et al., 2014). As a proof of concept, we tested whether the here proposed genetic profile predicted cortisol increase towards a psychosocial stress challenge.

Specific Aims and Hypotheses. The aims of the present study were twofold. (1) Build a genetic profile score that captures the disposition to greater HPA axis activity and determine its validity by testing it as a predictor of the endocrine stress response after a trigger. We hypothesized that the genetic profile score would positively predict cortisol increase towards a psychosocial stress challenge. (2) Investigate whether genetic variation in the HPA axis as captured by the GPS could explain inter-individual differences in facial emotion recognition impairments following early life stress. We hypothesized that the genetic profile score would moderate the relationship between ELS and performance in the FER task, such that for individuals carrying higher GPS the impact of ELS on facial emotion recognition in adult life is more severe. 
Hartling et al: Interaction of HPA axis genetics and early life stress shapes emotion recognition in healthy adults

\section{Methods}

Population. Two independent subsamples from a large-scale $(n=541)$ study conducted at Charité Universitätsmedizin Berlin were analysed for this study. The samples followed different protocols, and thus had different measures available. Sample 1 consisted of 64 participants who participated in a psychosocial stress challenge. Sample 2 consisted of 234 individuals who were presented a FER task. Sample characteristics are described in the results section and Supplementary Table 1. Inclusion criteria were age 18-90 years, absence of present and past diagnosis of psychiatric or neurologic disease, absence of major or unstable general medical conditions, and ability to participate in study procedures. Participants represent the middle range of socioeconomic status in the general population of Germany, and by self-report 100\% of participants in sample 1 and 2 are of European descent. Psychiatric disorders were screened for using the short version of the Structured Clinical Interview for Diagnostic and Statistical Manual of Mental Disorders (Wittchen et al., 1997). IQ was assessed with a German vocabulary test [Mehrfachwahl-Wortschatztest (Schmidt and Metzler, 1992)], an equivalent to the Nation Adult Reading Test). All subjects included in analyses had an IQ $>85$. The study was carried out in accordance with the latest version of the Declaration of Helsinki and approved by the Institutional Review Board of Charité Universitätsmedizin Berlin. All subjects gave written informed consent before screening and were reimbursed for participation.

Genotyping. DNA was extracted from whole blood. Subjects were genotyped for 14 SNPs in 3 genes coding for proteins relevant to the HPA axis (Supplementary Table 2) using iPLEX reagents and MassARRAY System (Sequenom Bioscience, San Diego, California). A locus-specific polymerase chain reaction amplification is followed by a single base extension of the SNP region performed by special primers. Subsequently, SNP alleles are identified with matrix-assisted laser desorption/ionisation time-offlight mass spectrometry (Gabriel and Ziaugra, 2004; Millis, 2011; Weissensteiner et al., 2013). Distribution of the SNPs studied here did not deviate significantly from Hardy-Weinberg-Equilibrium (pvalues are given in Table 1). All SNPs included in further analyses had a genotype missingness quota $<5 \%$ 
Hartling et al: Interaction of HPA axis genetics and early life stress shapes emotion recognition in healthy adults

and a minor allele frequency (MAF) of >5\%. FKBP 5 rs4713916 was dropped because of a missingness quota $>5 \%$.

Genetic profile score. We guided the construction of our genetic profile score by a previous approach of mapping genetic disposition to HPA axis activity based on prior evidence (Pagliaccio et al., 2014). Our array contained 3 of the 10 SNPs that were included therein (i.e. CRHR1 rs110402, FKBP5 rs1360780, NR3C1 rs41423247, marked in Table 1 with asterisks). We therefore included these 3 SNPs, maintaining the coding rules applied by Pagliaccio et al. (2014) for reasons of consistency.

We further added such SNPs from our array that have been functionally associated with differential HPA axis functioning in at least one peer-reviewed study. To grant that every SNP conveys independent information, only SNPs not in linkage disequilibrium (LD) were included as otherwise, the GPS would not linearly reflect genetic disposition. We tested for linkage disequilibrium with $\chi^{2}$-tests applying a significance level of $\mathrm{p}<.001$. rs10052957 on NR3C1, as well as rs3777747, rs3800373, and rs7757037 on FKBP5, were excluded because they were in LD with NR3C1 rs41423247 and FKBP5 rs1360780, respectively $\left(\chi^{2}(\mathrm{df}=4, \mathrm{~N}=370)>143.95 \mathrm{p}<.001\right)$. We identified $C R H R 1$ rs4792887, FKBP5 rs4713902 and NR3C1 rs6198 as variants matching these criteria. Hence, the GPS consisted of 6 SNPs in 3 genes (CRHR1 rs110402 and rs4792887, FKBP5 rs1360780 and rs4713902, and NR3C1 rs41423247 and rs6198). Supplementary Table 3 summarizes published evidence on the SNPs that were eventually included.

Across all loci, we assigned 1, 0.5 or 0 points to genotypes that are conferring of "strong" "intermediate" or "weak" disposition to a more reactive HPA axis based on previous evidence. Where it was available from the literature, we used information on the genetic model for the SNP (recessive, additive or dominant) to decide for the coding. If data on the genetic model was inconsistent or not available, we coded it to be additive. Table 1 shows these coding rules. Subsequently, scores were summed across loci resulting in the individual's HPA axis genetic profile score. GPS ranged from 0-6 with higher scores reflecting a genetic disposition to greater HPA axis activity. 
Hartling et al: Interaction of HPA axis genetics and early life stress shapes emotion recognition in healthy adults

\section{[Table 1]}

Montreal Imaging Stress Task \& Cortisol. Psychosocial stress was induced with the Montreal Imaging Stress Task (MIST; Dedovic et al., 2005). In the MIST, participants are shown mental arithmetic challenges while lying in the scanner. The items are chosen by an algorithm that varies task difficulty to yield a constant $45-50 \%$ correct response rate by adjusting complexity and time limit to the participant's performance. Feedback is given after each trial and a cumulative performance score is presented aside a fictive 'expected performance' score of $80 \%$. After half of the trials, participants are told that their performance is insufficient for their data to be useful and that they should hence please repeat the task with greater effort (i.e. take the parallel second half of the test). These elements of uncontrollability and social evaluative threat have been shown to induce behavioural and physiological stress and anxiety responses. After the session, subjects are debriefed that the task was designed to be impossible to accomplish and that it did not reflect their ability to perform mental arithmetic.

Saliva samples were collected using the Salivette sampling device (Sarstedt) while subjects were in the scanner before (T0) and immediately after the MIST task (T1, roughly 20min after T0; for a full description of the procedure, see Grimm et al., 2014). To ensure relatively stable endogenous cortisol levels, all subjects arrived between 1 and 3 p.m. in our laboratory. Saliva derived cortisol was analyzed using a time-resolved fluorescence immunoassay (Dressendörfer et al., 1992). We calculated the percentage of increase in saliva cortisol in response to the psychosocial stress challenge as follows: $\frac{T 1-T 0}{T 0} * 100$. In a recent study that extracted two components among the various cortisol measures used in psychoneuroendocrine research, this percentage increase measure was found to represent 'change in cortisol levels' (Khoury et al., 2015).

Early life stress assessment. A retrospective self-report measure, the German version of the Childhood Trauma Questionnaire (CTQ; Bernstein and Fink, 1998) was used to assess the extent of early life stress that subjects had experienced. The CTQ consists of 28 items (e.g. "When I grew up... ...people in my 
Hartling et al: Interaction of HPA axis genetics and early life stress shapes emotion recognition in healthy adults

family said hurtful or insulting things to me") on 5 subscales: physical abuse, physical neglect, emotional abuse, emotional neglect and sexual abuse. Scores on each subscale range from 5 to 25 , where higher scores indicate a more extensive exposure to that kind of stressful experience. A total score of $<29$ is considered as no exposure, scores of 29-34 as mild, of 34-41 as moderate and of 41 and above as severe (Scher et al., 2001).

Facial emotion recognition. We presented participants with 24 short video clips of target emotions (11 positive, 13 negative) portrayed by 15 professional actors ( 7 male, varying age (20-50 years)). In total, four basic (happy, disgusted, fearful, surprised) and 20 complex (interested, amused, aggrieved, troubled, jealous, enthusiastic, apologetic, disappointed, relieved, expectant, bored, compassionate, contemptuous, pardoning, embarrassed, wistful, furious, content, confident, doubtful) emotions were covered. The video clips were mute offering only visual cues. Subjects were seated in front of a screen, that showed the target video in the upper centre. Four emotion labels (one correct and three distractors) were provided in the lower half of the screen and participants were asked to choose the correct one. The trial was completed when the chosen label was dragged-and-dropped into a target area with the computer mouse. The task was self-paced, i.e. the video was repeated in loops until the trial was completed. Distractor labels consisted of (1) two emotions of the same valence, one with similar valence and arousal levels and one that differed more in arousal level but had the same valence as the target item, and (2) one emotion of the opposite valence. Figure 1 shows an example trial. Participants were asked to perform as fast and as accurately as possible. Performance was measured as the percentage of correct answers from all trials (For a more detailed report on task development and psychometric properties see "Face Puzzle explicit" in Kliemann et al., 2013)).

\section{[Figure 1 and caption]}

Statistical analysis. Covariate distribution was investigated with t-tests, contingency tables and Pearson or Spearman correlation for normally and non-normally distributed variables respectively. All descriptives are given as mean \pm standard deviation. All continuous values were mean-centred, and any interaction terms were 
Hartling et al: Interaction of HPA axis genetics and early life stress shapes emotion recognition in healthy adults

computed as the dot product of these. For display purposes, however, raw data values are used to aid interpretability. GPS main and interaction effects were tested with hierarchical multiple regression (HMR). For all regression analyses conducted, the number of predictor variables entered did not exceed the criterion of $n=20$ $+5 \mathrm{k}$ suggested by Khamis and Kepler (2010). We screened for multivariate outliers by calculating Mahalanobis distance prior to model estimation. Data points that exceeded a cut-off of $\mathrm{p}<.001$ for the $\chi^{2}$ value were excluded from analysis. We further identified data points that exerted an excessive influence on the HMR model, using Cook's distances following each model estimation. Data points with a Cook's distance exceeding the samplespecific cut-off of 4/sample size were removed from the analysis, and the HMR model was re-estimated. We report results from the final HMR models free of outliers and influential data points.

The association between GPS and cortisol increase to MIST was tested in an HMR model that in a first step contained factors know to predict cortisol response, age, gender and ELS exposure (Foley and Kirschbaum, 2010; Kudielka et al., 2004), and in the next step added genetic profile score as the predictor of interest. HMR was further employed to test whether the GPS and early life stress jointly predicted facial emotion recognition capacity. In the first step, three covariates that have previously associated with FER (Baker et al., 2014; Hall et al., 2010; Pardini and Nichelli, 2009; Sullivan and Ruffman, 2004) were entered: age, IQ, gender. In the second step, the genetic and environmental main predictors GPS and CTQ were entered together with interaction terms between covariates and main predictors (i.e. CTQ x gender, CTQ x age, CTQ x IQ, GPS x gender, GPS x age, GPS x IQ). This has been recommended in G x E interaction studies to better account for potential confounds (Keller, 2014). In the final step, the GPS x CTQ interaction term was entered. To examine the interaction effect, we tested whether successive regression steps significantly increased the variance explained by the model $\left(\Delta \mathrm{R}^{2}\right)$. We used the simple moderation model from the PROCESS tool for SPSS (Hayes, 2013) to probe significant interaction effects with the Johnson-Neyman technique (J-N technique), which determines significance transition points along the moderator. These values returned by the $\mathrm{J}-\mathrm{N}$ technique represent the range of the moderator within which the simple slope of the DV on the IV is significantly different from zero. 
Hartling et al: Interaction of HPA axis genetics and early life stress shapes emotion recognition in healthy adults

All tests were two-tailed, and the significance threshold was set at $\mathrm{p}<.05$. Statistical analyses were carried out using Predictive Analysis SoftWare, version 18.0.

\section{Results}

Hypothesis 1: Genetic profile score of HPA axis genes predicts cortisol response. 64 participants completed the MIST, 61 of which met the inclusion criteria. For 14 subjects, cortisol data was missing from T0 or T1 or both, and for another 6 genotyping was not successful for all 6 SNPs of interest (missing values were missing completely at random (MCAR), Little's MCAR test $\left.\chi^{2}(6, N=64)=11.162 \mathrm{p}=0.083\right)$. One subject was detected as a multivariate outlier, leaving a final sample of $n=40$ (18-62 years, $M=30.8 \pm 9.93$, $75 \%$ male). For each subject, a genetic profile score was computed as described above. The resulting GPS ranged from 0-4 and was normally distributed. Early life stress experience in this sample (i.e. CTQ range 25-68 M=35.68 \pm 10.92 ) was comparable to other community samples (Scher et al., 2001) and genetic profile score was not significantly associated with any of the covariates (i.e. age, gender, CTQ score; all p>.08). All correlations between variables and distribution between genders can be found in Supplementary Table 4.

A paired-samples t-test comparing raw salivary cortisol concentrations before MIST and after MIST in all individuals with cortisol measures from both time points $(n=50)$ indicated a significant increase in cortisol concentration from time point $\mathrm{t} 0(\mathrm{M}=3.37 \pm 3.35 \mathrm{nmol} / \mathrm{l})$ to $\mathrm{t} 1(\mathrm{M}=4.18 \pm 4.81 \mathrm{nmol} / \mathrm{l} ; \mathrm{t}(49)=-2.314 \mathrm{p}=.025)$. The percental increase in saliva cortisol ranged from $-68 \%$ to $+118 \%$ with $\mathrm{M}=11.2 \% \pm 55.9 \%$. 19 out of 40 participants $(47.5 \%)$ showed a positive increase in saliva cortisol, indicating they responded to the stress challenge (Pruessner et al., 2008). Within responders, the mean increase in cortisol levels was 53.2\% and was significant between $\mathrm{T} 0$ and $\mathrm{T} 1(\mathrm{Z}=-3.823 \mathrm{p}<.001)$. Supplementary Table 7 shows variables of interest compared between responders and non-responders. Responders' GPS ( $M=2.5 \pm 0.83)$ was significantly higher than non-responders' $(\mathrm{M}=1.93 \pm 0.93 ; \mathrm{t}(38)=-2.043 \mathrm{p}=.048)$. 
Hartling et al: Interaction of HPA axis genetics and early life stress shapes emotion recognition in healthy adults

A hierarchical regression model revealed GPS to be a significant predictor $\left(\beta=.42 \mathrm{p}=.008 \Delta \mathrm{R}^{2}=.17\right)$ of cortisol increase to psychosocial stress over and above the covariates age, gender and CTQ, all of which have previously been associated with cortisol response (Essex, 2011; Heim et al., 2004; Kudielka et al., 2004). The variance uniquely explained by genetic profile scores amounted to $17 \%\left(\Delta \mathrm{R}^{2}=.17, \mathrm{~F}(4,35)=\right.$ 7.928, $\mathrm{p}=.008$ ). All predictors' beta-weights and significance levels are presented in Table 2, aside to explained variance and F- and p-value for each step of the model individually and in change from the previous step. Figure 2 shows a scatter plot of cortisol increase in percent against genetic profile score with a simple regression line plotted.

\section{[Table 2 and Figure 2]}

Post-hoc, we excluded participants with a history of sexual abuse $(n=6)$ from the cortisol sample and ran the same hierarchical regression in that smaller sample $(n=34)$ as sexual abuse has been associated with distinct patterns of stress reactivity compared to other ELS subtypes (Essex, 2011). In this analysis too, genetic profile score significantly predicted cortisol increase $\left(\beta=-.298, \mathrm{p}=.028, \Delta \mathrm{R}^{2}=.144\right)$ above the covariates. It should be noted that this sample is smaller than recommended for multiple regression with this number of predictors.

Equally post-hoc, a two-step hierarchical multiple regression model (step 1: covariates age, gender and

CTQ; step 2: GPS) was calculated to predict baseline cortisol. Results did not show a significant relationship between the GPS and baseline cortisol $(\beta=0.042 \mathrm{p}=.267)$, indicating that the genetic association is specific to the triggered cortisol response. 
Hartling et al: Interaction of HPA axis genetics and early life stress shapes emotion recognition in healthy adults

\section{Hypothesis 2: Genetic profile score of HPA axis genes moderates the effect of early life stress}

on facial emotion recognition. 234 participants from the EEPT study were presented the FER task. As sexual abuse has been associated with distinct patterns of stress reactivity compared to other ELS subtypes (Essex, 2011), any reported sexual abuse experience led to exclusion from further analysis, which was the case for 34 subjects. We had to exclude 21 subjects for insufficient genetic data (missing values were missing completely at random, Little's $\operatorname{MCAR}$ test $\left.\chi^{2}(8, \mathrm{~N}=234)=14.472 \mathrm{p}=0.070\right)$, one multivariate outlier, and eight influential data points that exceeded the cut-off for Cook's distance. The remaining sample held $\mathrm{n}=170$ subjects (aged 19-77, M=43.2 \pm 19.9 years, 56\% male). CTQ total scores in this sample (i.e., $M=34.4 \pm 8.7$ ) were comparable to other community samples (Scher et al., 2001). Genetic profile scores ranged from 0 to 4.5 and were normally distributed (Supplementary Figure 2). GPS was not significantly associated with any of the covariates (all p>.19), all correlations between variables and distribution between genders can be found in Supplementary Table 5.

Accuracy in the facial emotion recognition task ranged from 50-95.8\% with $\mathrm{M}=78.3 \pm 9.5 \%$. A three-step hierarchical regression analysis was conducted to predict FER from genetic profile score and CTQ scores controlling for covariates age, gender and IQ, and possible interactions between covariates and the geneand environment variables. The model with all 12 predictors significantly explained $29.7 \%$ of the variance in facial emotion recognition (Model $\mathrm{R}^{2}=.297$, Model $\mathrm{R}_{\text {adj }}=.243, \mathrm{~F}(12,157)=5.525$, $\mathrm{p}<.001$ ). A simple effect of early life stress exposure reached marginal significance only in the third step of the analysis when the GPS-x-CTQ interaction term was added $(\beta=-.136, p=.056)$. Independently, genetic profile score did not significantly predict FER accuracy $(\beta=-.003, \mathrm{p}=.971)$. After accounting for all covariates, simple effects and gene-covariate- and environment-covariate-interactions, the interaction of CTQ and genetic profile score significantly predicted facial emotion recognition $(\beta=-.190, \mathrm{p}=.009)$. The interaction term of CTQ and GPS accounted for an additional $3.2 \%$ of explained variance $\left(\Delta \mathrm{R}^{2}=.032, \mathrm{~F}(1,157)=7.098, \mathrm{p}<.001\right)$. All predictors' beta-weights and significance levels are presented in Table 3, as well as explained variance and F- and p-value for each step of the model itself and in change from the previous step. 
Hartling et al: Interaction of HPA axis genetics and early life stress shapes emotion recognition in healthy adults

Post-hoc probation was done to understand the nature of the detected interaction. Johnson-Neyman testing revealed that CTQ scores were negatively associated with FER among those with a genetic profile score > 2.55 , i.e. the upper $54.2 \%$ of our sample. Figure 3 shows raw data (GPS plotted against task performance) overlain with model-derived simple slopes at different values for CTQ: mean - SD, mean, and mean $+\mathrm{SD}$. The shadowed area marks the region of significance where there is a significant negative effect of CTQ on facial emotion recognition. Supplementary Figure 3 visualises the region of significance of the interaction as the values of GPS where the confidence band of the conditional effect (the effect of CTQ at a certain value of the moderator GPS) does not include 0.

\section{[Table 3 and Figure 3]}

\section{Discussion}

The present study adopted a biologically-informed genetic profile score approach (Nikolova et al., 2011) to examine whether functionally relevant genetic variation within the HPA axis interacts with ELS to predict facial emotion recognition ability. Our results show that an additive genetic profile score of canonical HPA axis gene variants is predictive of the increase of cortisol towards a psychosocial stress challenges and moderates the negative effect that self-reported early life stress exerts on facial emotion recognition ability.

Genetic profile score of HPA axis genes predicts cortisol response. Genetic profile score positively predicted the increase of cortisol towards a psychosocial stress challenge. Individuals carrying a greater GPS and thus more variants linked to HPA axis function showed greater relative cortisol excretion when stressed by a mental arithmetic task. This result indicates that the GPS maps genetic disposition to a greater cortisol response to psychosocial stress, thereby providing validation for our polygenic approach. The GPS explained $17 \%$ of variance in the cortisol response to stress. Even though the rationale behind pooling genetic variability into profiles is to augment explanatory power, this effect size is surprisingly large. Even GWAS-derived polygenic risk scores, that comprise any genetic variants associated with 
Hartling et al: Interaction of HPA axis genetics and early life stress shapes emotion recognition in healthy adults

phenotype regardless of pathway, do not usually explain variance in this magnitude (with the exception of schizophrenia; Franke et al., 2016). In Pagliaccio and colleagues' study (2014) the HPA-axis polygenic score and stressful life events jointly explained $8 \%$ of variance. The present effect size should thus be considered an inflated estimate. Because of the moderate size of the cortisol sample we were unable to perform an analysis for an interaction effect between HPA axis genetic variation and early life stress shaping the cortisol response, although an already fairly rich literature suggests such effects for several of the variants included in our score (Mehta and Binder, 2012; Tyrka et al., 2009). Such an unaccounted-for interaction effect may make the simple effect seem larger than it truly is.

\section{Genetic profile score of HPA axis genes moderates the effect of early life stress on facial}

emotion recognition. As hypothesized, we found that the effect of ELS was moderated by the genetic profile score, such that the negative effect of ELS on facial emotion recognition was dependent on the number of stressresponsive genetic variants individuals carried. While individuals with lower GPS were not significantly affected by early life stress, we observed such an impact in individuals with a moderate to high GPS, with its severity increasing with GPS. The impact that exposure to early life stress exerts on an individual's affect recognition seems thus to be dependent on their disposition to HPA axis activity, where a less responsive make-up of the HPA axis is protective against ELS effects, while greater stress-responsivity is associated with greater malleability.

There is, to our knowledge, no previous behavioural study that has investigated a moderating role of HPA axis genes in the relationship between ELS and emotion recognition. However, several studies have investigated such a moderation in ELS-caused changes to the neural underpinnings of emotion processing. A recent report found an interaction between an HPA axis genetic profile score and CTQ scores predicting amygdala response to threat-related stimuli (Di Iorio et al., 2017). There, CTQ positively predicted amygdala reactivity only among individuals carrying more HPA axis variants, but no such association emerged for individuals with a lower or average number of genetic variants. Previously, two studies had shown one component of our genetic profile score, FKBP5 rs1360780, to be a moderator of the effect of 
Hartling et al: Interaction of HPA axis genetics and early life stress shapes emotion recognition in healthy adults

emotional neglect (a form of early life stress assessed with the CTQ) on amygdala responsivity towards emotional faces (Holz et al., 2015; White et al., 2012). To the contrary, Pagliaccio and colleagues (2015b) found a main effect of ELS on amygdala response to threat stimuli in children, but no significant moderation by a genetic profile of HPA axis activity. Notably, however, this study used a prospective instead of a retrospective measure of ELS. Several studies that employed both types of assessments have reported discrepant results where objective measures did not yield G x E-interaction effects while the retrospective CTQ did. This might be because retrospective measures capture emotionally loaded, memorable events that might be more predictive of ELS effects than acutely adverse family conditions.

Overall, these studies provide evidence that HPA axis genes moderate ELS effects on the processing of emotions. While the studies discussed above have only employed negative or threat-related stimuli, there is mixed evidence on valence-specific effects of early life stress effects on amygdala reactivity to emotional faces. Amygdala hyperactivity has often been found across emotional valence (McCrory et al., 2011; van Harmelen et al., 2013), yet sometimes only selectively for negative stimuli (Dannlowski et al., 2012). The amygdala was initially believed to be implicated in the processing of threat-related emotions exclusively, probably because its activation pattern is more pronounced for fearful stimuli. It has, however, since been associated with processing of both reward- and threat-related emotions (Leppänen and Nelson, 2009) and a recent meta-analysis confirmed greater amygdala responsivity to emotional stimuli across valences as an ecophenotype of ELS (Hein and Monk, 2017).

Similarly, a pattern of higher sensitivity and lower specificity of the amygdala has been found following acute stress in humans. Stress induction augments amygdala responses to equally high levels for threatrelated and positive stimuli, blurring the threat-selective activation pattern the amygdala showed in a control condition (van Marle et al., 2009). Reviewing ELS effects on amygdala and striatum, Fareri and Tottenham (2016) recently proposed that ELS impairs the amygdala's ability to feed value-based information into neural circuits associated with affective valuation. Although speculative at this point, these data provide a 
Hartling et al: Interaction of HPA axis genetics and early life stress shapes emotion recognition in healthy adults

potential mechanistic framework to understand how early environments influence subsequent emotion processing and the role of HPA axis genetics in this relationship. Further research with concurrent assessment of neural substrates and behavioural data of emotion recognition is needed to investigate this putative pathway.

There are a number of points that such a study could also help elucidate: For one, the relationship between FER and amygdala reactivity to threat that has been linked to genetic variants also outside the HPA axis (e.g. the serotonin transporter gene SLC6A4; Hariri et al., 2002) and is a manifestation, as well as, a risk factor of mood and anxiety disorders (Avinun et al., 2017). Further, the role of structures apart from the amygdala, that are involved in facial emotion recognition (Fusar-Poli et al., 2009) and architecturally or functionally changed by chronic stress (Arnsten, 2015; Teicher et al., 2016) but have thus far received less attention (Hein and Monk, 2017). For conceptual clarity, FER deficits following ELS should be disentangled from alexithymia, a personality trait characterized by difficulties identifying and communicating subjective feelings, that has been equally linked to early life stress (Aust et al., 2013; Terock et al., 2018)). Subjects with high levels of alexithymia have been shown to have difficulties not only naming their own but also inferring emotions from other people's facial expressions (Grynberg et al., 2012) and show reduced volume of brain regions that are involved in FER (Xu et al., 2018).

From the data available, it cannot be ruled that the interaction of HPA axis genes with CTQ scores is not mediated by adult life functioning of the HPA axis. However, previous studies found no influence of acute cortisol levels on facial emotion recognition (Smeets et al., 2009). It is thus unlikely that acute cortisol levels during the task had an influence on facial emotion recognition.

It should be noted that the simple effect of CTQ on facial emotion recognition did not reach significance. As a simple effect in HMR models with mean-centred variables and an interaction effect it can be interpreted as the sole effect of CTQ when the HPA profile is set to average as well as all covariates set to their mean, which would have been predicted from previous reports. This points to a generally rather 
Hartling et al: Interaction of HPA axis genetics and early life stress shapes emotion recognition in healthy adults

weak association between exposure to early life stress and FER ability in a population of healthy adults. This is plausible in the light of studies showing that deficits in FER after early life stress are remediable (Bick and Nelson, 2016; Nelson et al., 2013).

The interaction of CTQ scores and genetic profile significantly explained an additional $3.2 \%$ of variance in facial emotion recognition after accounting for all covariates and potential confounds. This is a considerable effect size compared to single SNP studies (Manolio et al., 2009). Thus, this study provides novel evidence for the utility of biologically-informed genetic profile scores in mapping interindividual differences in susceptibility to ELS effects.

Limitations. It is important to consider the present results in the context of the limitations of the polygenic profile approach, the cross-sectional study design, sample size and the large-scale study approach. While combining several sources of genetic variability reflecting one biological trait has several strengths like the increase in power and literature-derived composition, it is also a relatively novel approach that is faced with challenges. Biologically informed polygenic approaches rely essentially on prior functional association studies, which have sometimes been inconsistent and still need more replication. Notably, for the CRHRI variant rs110402, both the A- and G-allele have been linked to indices of greater HPA axis reactivity (Cicchetti et al., 2011; Mahon et al., 2013). Further, only two of the included variants, FKBP5 rs167830 and NR3C1 rs6198, have been functionally characterised, while the remaining SNPs are intronic and there is, to date, no evidence of their mechanistic relevance. Henceforth, the functional relevance of our score still needs to be explored. These caveats notwithstanding, the association between the genetic profile score we propose and the cortisol response to psychosocial stress can be regarded as a proof of concept, indicating that the GPS reflects genetic disposition towards a stronger cortisol response.

In addition, collapsing genetic variability relies on several assumptions. Firstly, supposing additivity, hence a linear relationship between SNPs and phenotype, it neglects potential epistatic (i.e. gene-gene-interaction) effects. This may be regarded a strong assumption, although there is some support for this view in complex 
Hartling et al: Interaction of HPA axis genetics and early life stress shapes emotion recognition in healthy adults

trait genetics (Polderman et al., 2015). Secondly, it neglects potential downstream homeostatic regulations (e.g. receptor downregulation). Thirdly, SNPs are unlikely to contribute equally to the phenotype across different genes, as using an unweighted score implies. However, ample, and ideally meta-analytic, data is needed to derive individual SNPs' weights, which is not yet available from the literature. Refining the polygenic profile approach is an important purpose for future research that will be vastly helped by better priors, comprehensive understanding of biological pathways, and meta-analytically determined effect sizes. To aid this in the future, we have presented the effects of individual SNPs in the supplement (Supplementary Table 6).

While we sought to follow methods of GPS construction by another group (Pagliaccio et al., 2014), we were unable to test the exact same genetic profile because of insufficient genetic data and thus created a new one applying their selection criteria on our dataset. Following a different selection rationale (including only functionally characterized variants into the score), Dilorio and colleagues (2017) tested yet another genetic profile of HPA axis genetic variability. This diversity of approaches (emerging from different selection criteria and coding rules) illustrates that, at this point, there are a plethora of reasonable polygenic scores that could be employed. This increases the risk of false positives and impedes meta-analysis on this kind of study. Moving forward, the field should strive to develop standard profiles for different pathways as research tools.

There are also some limitations to the study design that need to be considered. First, while the task employed here is an especially naturalistic measure of facial emotion recognition, due to its employment of video material of complex emotions, it prohibits conclusions on the differential recognition of basic emotions and we can thus not relate our results to the body of literature that investigated such effects (Pollak et al., 2000). Secondly, as we did not directly assess amygdala reactivity, we can only speculate about the neural mechanisms that mediate the interaction effect we observed. Further, the cross-sectional design of this study prohibits the analysis of the genetic disposition, environmental stressors, and FER within a developmental 
Hartling et al: Interaction of HPA axis genetics and early life stress shapes emotion recognition in healthy adults

framework. Future research in a longitudinal sample that also integrates biomarker assessments could elucidate the developmental trajectories and endophenotypes that lead toward adult affective processing. Thirdly, the CTQ is a global measure of stressful situations collapsed over the first 17 years of life that does not assess factors like timing and chronicity of the stressor, which likely shape ELS effects (Lupien et al., 2009). Future studies would ideally employ a more fine-grained analysis of the environmental variable. Studying early life stress effects in healthy adults also comes with the risk of disproportionally sampling resilient individuals. Further, our study used a design regressing over the continuum of stressful experiences in childhood, from exposure in the normal range to cases of harsh maltreatment, with only $16 \%$ of our sample falling into the 'severe' category of ELS. While this approach is helpful in detecting gradual alterations in neurocognitive functioning as a function of the extent of ELS exposure, it does not allow for the same strong inferences about consequences of severe maltreatment that an exposed-control group design would.

Moreover, given that genetic effects are known to be small and oftentimes hard to replicate (Avinun et al., 2017) the sample size is a major limitation of this study. This is especially true for the smaller sample of the cortisol study, as cortisol is in itself a noisy measure (Kudielka et al., 2009). Further, a substantial number of participants values (20 out of 64 in sample 1, 21 out of 234 in sample 2, i.e. $>5 \%$ ) could not be included in analyses due to missing values from laboratory analyses. The present report should thus be treated with caution until replicated in an independent sample.

More broadly, it should also be noted that this study was part of a large-scale investigation into the interactive effects of early environment and genetics in shaping emotional phenotypes. This type of research allows for the compilation of a well-powered dataset that can be used to address multiple research questions, an asset especially for studies with expectedly small effect sizes like genetic effects. However, such studies often lack some data that would be useful for specific research questions (in the case of this study, for example, $N R 3 C 2$ variants, that have been used in previous genetic profiles of the HPA axis (Di Iorio et al., 
Hartling et al: Interaction of HPA axis genetics and early life stress shapes emotion recognition in healthy adults

2017; Pagliaccio et al., 2014), were not available from the dataset) or rely on easily assessable measures (such as the CTQ instead of more fine-grained and objective ELS assessments and cortisol rather than ACTH assessment), which are obvious limitations. Finally, with the possibility to test multiple hypotheses comes higher risk for false positives. Ultimately, this can only be alleviated by transparency regarding (and possibly also formal pre-registration of) hypotheses and planned analyses and, crucially, replication.

\section{Conclusion and future directions}

Limitations notwithstanding, this study provides evidence suggesting that a profile of genetic variance associated with variability in HPA axis activity moderates the impact of early life stress on facial emotion recognition. If replicated in an independent sample, the effect described here may indicate a biological mechanism of vulnerability towards the detrimental effects that ELS has on social and emotional functioning. The present study contributes to a growing body of literature emphasising the importance of this pathway in understanding ELS effects.

Several interesting directions for future research emerge from this study. First, further investigation into the interplay of molecular, endocrine and brain functional and behavioural levels of early life stress effects will shed further light on the developmental trajectories that shape normal and vulnerable patterns of responding to social cues of emotions. An emerging line of research suggests that gene methylation of HPA axis related genes may mediate early life stress effects (Bogdan et al., 2016; Romens et al., 2015). Concurrent assessment of genotype and methylation rate in studies on ELS effects will shed further light on this putative pathway. Second, to understand better the normative and impaired functioning of the amygdala, an important goal for future studies is to investigate amygdala responsiveness towards, and its role in, the recognition of both threat- and reward-related emotional stimuli. Third, investigating how ELS-caused deficits in facial emotion recognition are related to broader difficulties with emotion processing and regulation or confer vulnerability to affective and social disorders will inform preventive and therapeutic 
Hartling et al: Interaction of HPA axis genetics and early life stress shapes emotion recognition in healthy adults

interventions. There is promising evidence that early intervention can support recovery from damage done to emotion-related brain circuits caused by early life stress (Bick and Nelson, 2016).

More broadly, our study adds to the emerging evidence that genetic profiles curated to capture the systemlevel genetic variation of biological traits may become powerful research tools that will advance the study of individual differences in risk and resilience. 
Hartling et al: Interaction of HPA axis genetics and early life stress shapes emotion recognition in healthy

adults

\section{References}

Arnsten, A.F.T., 2015. Stress weakens prefrontal networks: Molecular insults to higher cognition. Nat. Neurosci. 18, 1376-1385. https://doi.org/10.1038/nn.4087

Aust, S., Härtwig, E.A., Heuser, I., Bajbouj, M., 2013. The role of early emotional neglect in alexithymia. Psychol. Trauma Theory, Res. Pract. Policy 5, 225-232. https://doi.org/10.1037/a0027314

Avinun, R., Nevo, A., Knodt, A.R., Elliott, M.L., Hariri, A.R., 2017. Replication in imaging genetics: The case of threat-related amygdala reactivity. Biol. Psychiatry. https://doi.org/10.1016/j.biopsych.2017.11.010

Baker, C.A., Peterson, E., Pulos, S., Kirkland, R.A., 2014. Eyes and IQ: A meta-analysis of the relationship between intelligence and "Reading the Mind in the Eyes." Intelligence 44, 78-92. https://doi.org/10.1016/j.intell.2014.03.001

Bernstein, D.P., Fink, L., 1998. Childhood trauma questionnaire: A retrospective self-report: Manual. Psychological Corporation.

Bick, J., Nelson, C.A., 2016. Early adverse experiences and the developing brain. Neuropsychopharmacology 41, 177-196. https://doi.org/10.1038/npp.2015.252

Bogdan, R., Pagliaccio, D., Baranger, D.A., Hariri, A.R., 2016. Genetic moderation of stress effects on corticolimbic circuitry. Neuropsychopharmacology 41, 275-296. https://doi.org/10.1038/npp.2015.216

Cicchetti, D., Rogosch, F.A., Oshri, A., 2011. Interactive effects of corticotropin releasing hormone receptor 1, serotonin transporter linked polymorphic region, and child maltreatment on diurnal cortisol regulation and internalizing symptomatology. Dev. Psychopathol. 23, 1125-1138. https://doi.org/10.1017/S0954579411000599

da Silva Ferreira, G.C., Crippa, J.A.S., de Lima Osorio, F., 2014. Facial emotion processing and recognition among maltreated children: A systematic literature review. Front. Psychol. 5, 1-10. https://doi.org/10.3389/fpsyg.2014.01460

Dannlowski, U., Stuhrmann, A., Beutelmann, V., Zwanzger, P., Lenzen, T., Grotegerd, D., Domschke, K., Hohoff, C., Ohrmann, P., Bauer, J., Lindner, C., Postert, C., Konrad, C., Arolt, V., Heindel, W., Suslow, T., Kugel, H., 2012. Limbic scars: Long-term consequences of childhood maltreatment revealed by functional and structural magnetic resonance imaging. Biol. Psychiatry 71, 286-293. https://doi.org/10.1016/j.biopsych.2011.10.021

Dedovic, K., Renwick, R., Mahani, N.K., Engert, V., Lupien, S.J., Pruessner, J.C., 2005. The Montreal Imaging Stress Task: Using functional imaging to investigate the effects of perceiving and processing psychosocial stress in the human brain, in: Journal of Psychiatry and Neuroscience. pp. 319-325.

DeRijk, R.H., 2009. Single nucleotide polymorphisms related to HPA axis reactivity. Neuroimmunomodulation 16, 340-352. https://doi.org/10.1159/000216192

Di Iorio, C.R., Carey, C.E., Michalski, L.J., Corral-Frias, N.S., Conley, E.D., Hariri, A.R., Bogdan, R., 2017. Hypothalamic-pituitary-adrenal axis genetic variation and early stress moderates amygdala function. Psychoneuroendocrinology 80, 170-178. https://doi.org/10.1016/j.psyneuen.2017.03.016

Dressendörfer, R.A., Kirschbaum, C., Rohde, W., Stahl, F., Strasburger, C.J., 1992. Synthesis of a cortisol-biotin conjugate and evaluation as a tracer in an immunoassay for salivary cortisol measurement. J. Steroid Biochem. Mol. Biol. 43, 683-692.

Essex, M.J., 2011. Influence of early life stress on later hypothalamic-pituitary-adrenal axis functioning and its covariation with mental health sympotoms: A study of the allostatic process from childhood into adolescence. Dev. Psychopathol. 23, 1039-1058. https://doi.org/doi:10.1017/S0954579411000484

Fareri, D.S., Tottenham, N., 2016. Effects of early life stress on amygdala and striatal development. Dev. Cogn. Neurosci. 19, 233-247. https://doi.org/10.1016/j.den.2016.04.005 
Hartling et al: Interaction of HPA axis genetics and early life stress shapes emotion recognition in healthy

adults

Federenko, I.S., Nagamine, M., Hellhammer, D.H., Wadhwa, P.D., Wüst, S., 2004. The heritability of hypothalamus pituitary adrenal axis responses to psychosocial stress is context dependent. J. Clin. Endocrinol. Metab. 89, 6244-6250. https://doi.org/10.1210/jc.2004-0981

Foley, P., Kirschbaum, C., 2010. Human hypothalamus-pituitary-adrenal axis responses to acute psychosocial stress in laboratory settings. Neurosci. Biobehav. Rev. 35, 91-96. https://doi.org/10.1016/j.neubiorev.2010.01.010

Franke, B., Stein, J.L., Ripke, S., Anttila, V., Hibar, D.P., van Hulzen, K.J.E., Arias-Vasquez, A., Smoller, J.W., Nichols, T.E., Neale, M.C., McIntosh, A.M., Lee, P., McMahon, F.J., Meyer-Lindenberg, A., Mattheisen, M., Andreassen, O.A., Gruber, O., Sachdev, P.S., Roiz-Santiañez, R., Saykin, A.J., Ehrlich, S., Mather, K.A., Turner, J.A., Schwarz, E., Thalamuthu, A., Yao, Y., Ho, Y.Y.W., Martin, N.G., Wright, M.J., O’Donovan, M.C., Thompson, P.M., Neale, B.M., Medland, S.E., Sullivan, P.F., Medland, S.E., Sullivan, P.F., 2016. Genetic influences on schizophrenia and subcortical brain volumes: large-scale proof of concept. Nat. Neurosci. 19, 420-431. https://doi.org/10.1038/nn.4228

Fusar-Poli, P., Placentino, A., Carletti, F., Landi, P., Allen, P., Surguladze, S., Benedetti, F., Abbamonte, M., Gasparotti, R., Barale, F., Perez, J., McGuire, P., Politi, P., 2009. Functional atlas of emotional faces processing: A voxel-based meta-analysis of 105 functional magnetic resonance imaging studies. J. Psychiatry Neurosci. 34, 418-432. https://doi.org/10.1016/S1180-4882(09)50077-7

Gabriel, S., Ziaugra, L., 2004. SNP Genotyping using Sequenom MassARRAY 7K Platform, in: Current Protocols in Human Genetics. Wiley Online Library, pp. 2-12. https://doi.org/10.1002/0471142905.hg0212s42

Germine, L., Dunn, E.C., Mclaughlin, K.A., Smoller, J.W., 2015. Childhood adversity is associated with adult theory of mind and social affiliation, but not face processing. PLoS One 10, e0129612. https://doi.org/10.1371/journal.pone.0129612

Grimm, S., Pestke, K., Feeser, M., Aust, S., Weigand, A., Wang, J., Wingenfeld, K., Pruessner, J.C., La Marca, R., Böker, H., Bajbouj, M., 2014. Early life stress modulates oxytocin effects on limbic system during acute psychosocial stress. Soc. Cogn. Affect. Neurosci. 9, 1828-1835. https://doi.org/10.1093/scan/nsu020

Grynberg, D., Chang, B., Corneille, O., Maurage, P., Vermeulen, N., Berthoz, S., Luminet, O., 2012. Alexithymia and the processing of emotional facial expressions (EFEs): Systematic review, unanswered questions and further perspectives. PLoS One 7. https://doi.org/10.1371/journal.pone.0042429

Hall, J.K., Hutton, S.B., Morgan, M.J., 2010. Sex differences in scanning faces: Does attention to the eyes explain female superiority in facial expression recognition? Cogn. Emot. 24, 629-637. https://doi.org/10.1080/02699930902906882

Hariri, A.R., Mattay, V.S., Tessitore, A., Kolachana, B., Fera, F., Goldman, D., Egan, M.F., Weinberger, D.R., 2002. Serotonin Transporter Genetic Variation and the Response of the Human Amygdala. Science (80-. ). 297, 400-403. https://doi.org/10.1126/science.1071829

Hayes, A.F., 2013. Introduction to mediation, moderation, and conditional process analysis: A regression-based approach. Guilford Press.

Heim, C., Newport, D.J., Bonsall, R., Miller, A.H., Nemeroff, C.B., 2004. Altered pituitary-adrenal axis responses to provocative challenge tests in adult survivors of childhood abuse. Am. J. Psychiatry 158, 282-289. https://doi.org/10.1016/S0306-4530(02)00146-4

Hein, T.C., Monk, C.S., 2017. Research Review: Neural response to threat in children, adolescents, and adults after child maltreatment - a quantitative meta-analysis. J. Child Psychol. Psychiatry Allied Discip. 58, 222-230. https://doi.org/10.1111/jcpp.12651

Holz, N.E., Buchmann, A.F., Boecker, R., Blomeyer, D., Baumeister, S., Wolf, I., Rietschel, M., Witt, S.H., Plichta, M.M., Meyer-Lindenberg, A., Banaschewski, T., Brandeis, D., Laucht, M., Tobias, A.M., Daniel, B., Manfred, B., Meyer-Lindenberg, A., Banaschewski, T., Brandeis, D., Laucht, M., 2015. Role of FKBP5 in emotion processing: results on amygdala activity, connectivity and volume. Brain Struct. Funct. 220, 1355- 
Hartling et al: Interaction of HPA axis genetics and early life stress shapes emotion recognition in healthy

adults

1368. https://doi.org/10.1007/s00429-014-0729-5

Jedd, K., Hunt, R.H., Cicchetti, D., Hunt, E., Cowell, R.A., Rogosch, F.A., Toth, S.L., Thomas, K.M., 2015. Longterm consequences of childhood maltreatment: Altered amygdala functional connectivity. Dev. Psychopathol. 27, 1577-1589. https://doi.org/10.1017/S0954579415000954

Keller, M.C., 2014. Gene $\times$ environment interaction studies have not properly controlled for potential confounders: The problem and the (simple) solution. Biol. Psychiatry 75, 18-24.

https://doi.org/10.1016/j.biopsych.2013.09.006

Khamis, H.J., Kepler, M., 2010. Sample size in multiple regression: 20+ 5K. J. Appl. Stat. Sci. 17, 505.

Khoury, J.E., Gonzalez, A., Levitan, R.D., Pruessner, J.C., Chopra, K., Basile, V.S., Masellis, M., Goodwill, A., Atkinson, L., 2015. Summary cortisol reactivity indicators: Interrelations and meaning. Neurobiol. Stress 2, 34-43. https://doi.org/10.1016/j.ynstr.2015.04.002

Kliemann, D., Rosenblau, G., Bölte, S., Heekeren, H.R., Dziobek, I., 2013. Face puzzle-two new video-based tasks for measuring explicit and implicit aspects of facial emotion recognition. Front. Psychol. 4, 1-13. https://doi.org/10.3389/fpsyg.2013.00376

Kudielka, B.M., Buske-Kirschbaum, A., Hellhammer, D.H., Kirschbaum, C., 2004. HPA axis responses to laboratory psychosocial stress in healthy elderly adults, younger adults, and children: Impact of age and gender. Psychoneuroendocrinology 29, 83-98. https://doi.org/10.1016/S0306-4530(02)00146-4

Kudielka, B.M., Hellhammer, D.H., Wüst, S., 2009. Why do we respond so differently? Reviewing determinants of human salivary cortisol responses to challenge. Psychoneuroendocrinology 34, 2-18.

https://doi.org/10.1016/j.psyneuen.2008.10.004

Leppänen, J.M., Nelson, C.A., 2009. Tuning the developing brain to social signals of emotions. Nat. Rev. Neurosci. $10,37-47$.

Lupien, S.J., McEwen, B.S., Gunnar, M.R., Heim, C., 2009. Effects of stress throughout the lifespan on the brain, behaviour and cognition. Nat. Rev. Neurosci. 10, 434-445. https://doi.org/10.1038/nrn2639

Mahon, P.B., Zandi, P.P., Potash, J.B., Nestadt, G., Wand, G.S., 2013. Genetic association of FKBP5 and CRHR1 with cortisol response to acute psychosocial stress in healthy adults. Psychopharmacology (Berl). 227, 231241. https://doi.org/10.1007/s00213-012-2956-X

Manolio, T.A., Collins, F.S., Cox, N.J., Goldstein, D.B., Hindorff, L.A., Hunter, D.J., McCarthy, M.I., Ramos, E.M., Cardon, L.R., Chakravarti, A., Cho, J.H., Guttmacher, A.E., Kong, A., Kruglyak, L., Mardis, E., Rotimi, C.N., Slatkin, M., Valle, D., Whittemore, A.S., Boehnke, M., Clark, A.G., Eichler, E.E., Gibson, G., Haines, J.L., MacKay, T.F.C., McCarroll, S.A., Visscher, P.M., 2009. Finding the missing heritability of complex diseases. Nature 461, 747-753. https://doi.org/10.1038/nature08494

McCrory, E.J., De Brito, S.A., Sebastian, C.L., Mechelli, A., Bird, G., Kelly, P.A., Viding, E., 2011. Heightened neural reactivity to threat in child victims of family violence. Curr. Biol.

https://doi.org/10.1016/j.cub.2011.10.015

Mehta, D., Binder, E.B., 2012. Gene × environment vulnerability factors for PTSD: The HPA-axis. Neuropharmacology. https://doi.org/10.1016/j.neuropharm.2011.03.009

Millis, M.P., 2011. Medium-throughput SNP genotyping using mass spectrometry: multiplex SNP genotyping using the iPLEX(R) Gold assay. Methods Mol. Biol. 700, 61-76. https://doi.org/10.1007/978-1-61737-954-3_5

Myers, B., McKlveen, J.M., Herman, J.P., 2014. Glucocorticoid actions on synapses, circuits, and behavior: Implications for the energetics of stress. Front. Neuroendocrinol. 35, 180-196. https://doi.org/10.1016/j.yfrne.2013.12.003

Nelson, C.A., Westerlund, A., McDermott, J.M., Zeanah, C.H., Fox, N.A., 2013. Emotion recognition following 
Hartling et al: Interaction of HPA axis genetics and early life stress shapes emotion recognition in healthy

adults

early psychosocial deprivation. Dev. Psychopathol. 25, 517-525. https://doi.org/10.1017/S0954579412001216

Nikolova, Y.S., Ferrell, R.E., Manuck, S.B., Hariri, A.R., 2011. Multilocus genetic profile for dopamine signaling predicts ventral striatum reactivity. Neuropsychopharmacology 36, 1940-7.

https://doi.org/10.1038/npp.2011.82

Pagliaccio, D., Luby, J.L., Bogdan, R., Agrawal, A., Gaffrey, M.S., Belden, A.C., Botteron, K.N., Harms, M.P., Barch, D.M., 2015a. Amygdala functional connectivity, HPA axis genetic variation, and life stress in children and relations to anxiety and emotion regulation. J. Abnorm. Psychol. 124, 817-833. https://doi.org/10.1037/abn0000094

Pagliaccio, D., Luby, J.L., Bogdan, R., Agrawal, A., Gaffrey, M.S., Belden, A.C., Botteron, K.N., Harms, M.P., Barch, D.M., 2015b. HPA axis genetic variation, pubertal status, and sex interact to predict amygdala and hippocampus responses to negative emotional faces in school-age children. Neuroimage 109, 1-11. https://doi.org/10.1016/j.neuroimage.2015.01.017

Pagliaccio, D., Luby, J.L., Bogdan, R., Agrawal, A., Gaffrey, M.S., Belden, A.C., Botteron, K.N., Harms, M.P., Barch, D.M., 2014. Stress-system genes and life stress predict cortisol levels and amygdala and hippocampal volumes in children. Neuropsychopharmacology 39, 1245-1253. https://doi.org/10.1038/npp.2013.327

Pardini, M., Nichelli, P.F., 2009. Age-related decline in mentalizing skills across adult life span. Exp. Aging Res. 35, 98-106. https://doi.org/10.1080/03610730802545259

Polderman, T.J.C., Benyamin, B., De Leeuw, C.A., Sullivan, P.F., Van Bochoven, A., Visscher, P.M., Posthuma, D., 2015. Meta-analysis of the heritability of human traits based on fifty years of twin studies. Nat. Genet. 47, 702-709.

Pollak, S.D., Cicchetti, D., Hornung, K., Reed, A., 2000. Recognizing emotion in faces: developmental effects of child abuse and neglect. Dev. Psychol. 36, 679-688. https://doi.org/10.1037//0012-1649.36.5.679

Pruessner, J.C., Dedovic, K., Khalili-Mahani, N., Engert, V., Pruessner, M., Buss, C., Renwick, R., Dagher, A., Meaney, M.J., Lupien, S., 2008. Deactivation of the limbic system during acute psychosocial stress: Evidence from positron emission tomography and functional magnetic resonance imaging studies. Biol. Psychiatry 63, 234-240. https://doi.org/10.1016/j.biopsych.2007.04.041

Romens, S.E., McDonald, J., Svaren, J., Pollak, S.D., 2015. Associations between early life stress and gene methylation in children. Child Dev. 86, 303-309. https://doi.org/10.1111/cdev.12270

Scher, C.D., Stein, M.B., Asmundson, G.J.G., Mccreary, D.R., Forde, D.R., 2001. The Childhood Trauma Questionnaire in a community sample : Psychometric properties and normative data 14.

Schmidt, K.-H., Metzler, P., 1992. Wortschatztest: WST. Beltz.

Smeets, T., Dziobek, I., Wolf, O.T., 2009. Social cognition under stress: differential effects of stress-induced cortisol elevations in healthy young men and women. Horm. Behav. 55, 507-513.

Sullivan, S., Ruffman, T., 2004. Emotion recognition deficits in the elderly. Int. J. Neurosci. 114, 403-432. https://doi.org/10.1080/00207450490270901

Teicher, M.H., Samson, J.A., Anderson, C.M., Ohashi, K., 2016. The effects of childhood maltreatment on brain structure, function and connectivity. Nat. Rev. Neurosci. 17, 652-66. https://doi.org/10.1038/nrn.2016.111

Terock, J., Van Der Auwera, S., Janowitz, D., Homuth, G., Hannemann, A., Schmidt, C.O., Meyer Zu Schwabedissen, H., Freyberger, H.J., Grabe, H.J., 2018. Childhood trauma and functional variants of 5HTTLPR are independently associated with alexithymia in 5,283 subjects from the general population. Psychother. Psychosom. 87, 58-61. https://doi.org/10.1159/000484143

Tyrka, A.R., Price, L.H., Gelernter, J., Schepker, C., Anderson, G.M., Carpenter, L.L., 2009. Interaction of childhood maltreatment with the corticotropin-releasing hormone receptor gene: Effects on hypothalamic- 
Hartling et al: Interaction of HPA axis genetics and early life stress shapes emotion recognition in healthy adults

pituitary-adrenal axis reactivity. Biol. Psychiatry 66, 681-685. https://doi.org/10.1016/j.biopsych.2009.05.012

van Harmelen, A.-L., van Tol, M.-J., Demenescu, L.R., van der Wee, N.J.A., Veltman, D.J., Aleman, A., van Buchem, M.A., Spinhoven, P., Penninx, B.W.J.H., Elzinga, B.M., 2013. Enhanced amygdala reactivity to emotional faces in adults reporting childhood emotional maltreatment. Soc. Cogn. Affect. Neurosci. 8, 362369.

van Marle, H.J.F., Hermans, E.J., Qin, S., Fernández, G., 2009. From specificity to sensitivity: How acute stress affects amygdala processing of biologically salient stimuli. Biol. Psychiatry 66, 649-655. https://doi.org/10.1016/j.biopsych.2009.05.014

Weissensteiner, H., Haun, M., Schönherr, S., Neuner, M., Forer, L., Specht, G., Kloss-Brandstätter, A., Kronenberg, F., Coassin, S., 2013. SNPflow: A lightweight application for the processing, storing and automatic quality checking of genotyping assays. PLoS One 8, e59508. https://doi.org/10.1371/journal.pone.0059508

White, M.G., Bogdan, R., Fisher, P.M., Muñoz, K.E., Williamson, D.E., Hariri, A.R., 2012. FKBP5 and emotional neglect interact to predict individual differences in amygdala reactivity. Genes, Brain Behav. 11, 869-878. https://doi.org/10.1111/j.1601-183X.2012.00837.x

Wittchen, H.-U., Zaudig, M., Fydrich, T., 1997. Strukturiertes Klinisches Interview für DSM-IV. Hogrefe, Göttingen.

Xu, P., Opmeer, E.M., van Tol, M.J., Goerlich, K.S., Aleman, A., 2018. Structure of the alexithymic brain: A parametric coordinate-based meta-analysis. Neurosci. Biobehav. Rev. 87, 50-55.

https://doi.org/10.1016/j.neubiorev.2018.01.004 
Hartling et al: Interaction of HPA axis genetics and early life stress shapes emotion recognition in healthy adults

Figr-1

\section{Übungsdurchgang}

Ordnen Sie dem Gesicht die passende Emotion zu.

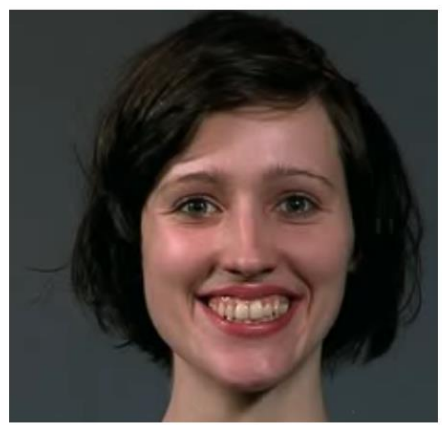

1. heiter

2. stolz

3. ängstlich

4. verliebt

Figure 1 Screenshot of a sample video stimulus of the facial emotion recogntion task and 4 response options. The target response 'carefree' ['heiter'] is presented among 3 distractors, one with the same valence and arousal level ('proud'['stolz']), one with the same valence but different arousal level ('in love'['verliebt']) and one emotion of the opposite valence ('scared'['ängstlich']l). 
Hartling et al: Interaction of HPA axis genetics and early life stress shapes emotion recognition in healthy adults

Figr-2

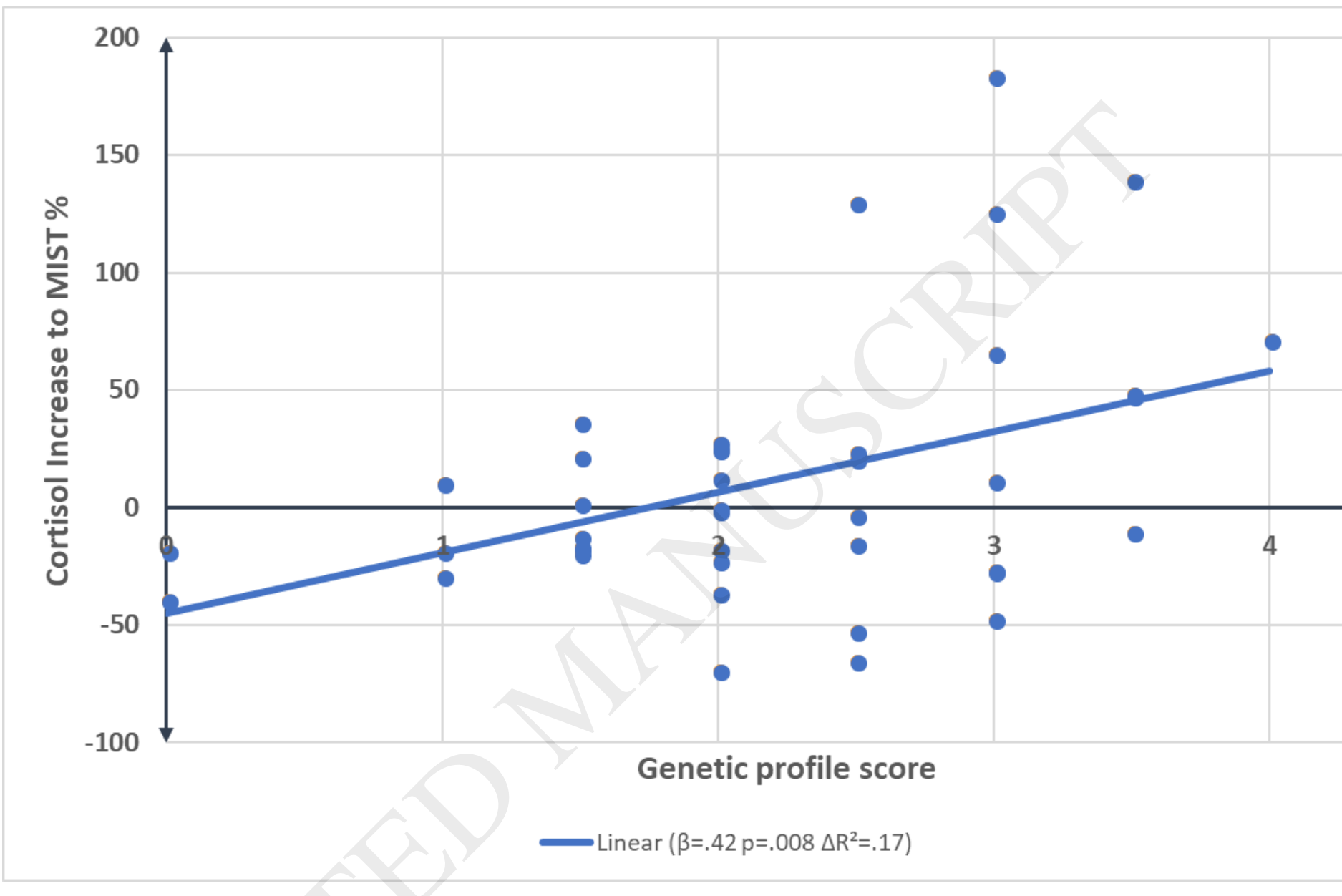

Figure 2 Percentual cortisol increase to psychosocial stress challenge (Montreal Imaging Stress Test) plotted against genetic profile score. 
Hartling et al: Interaction of HPA axis genetics and early life stress shapes emotion recognition in healthy adults

Figr-3

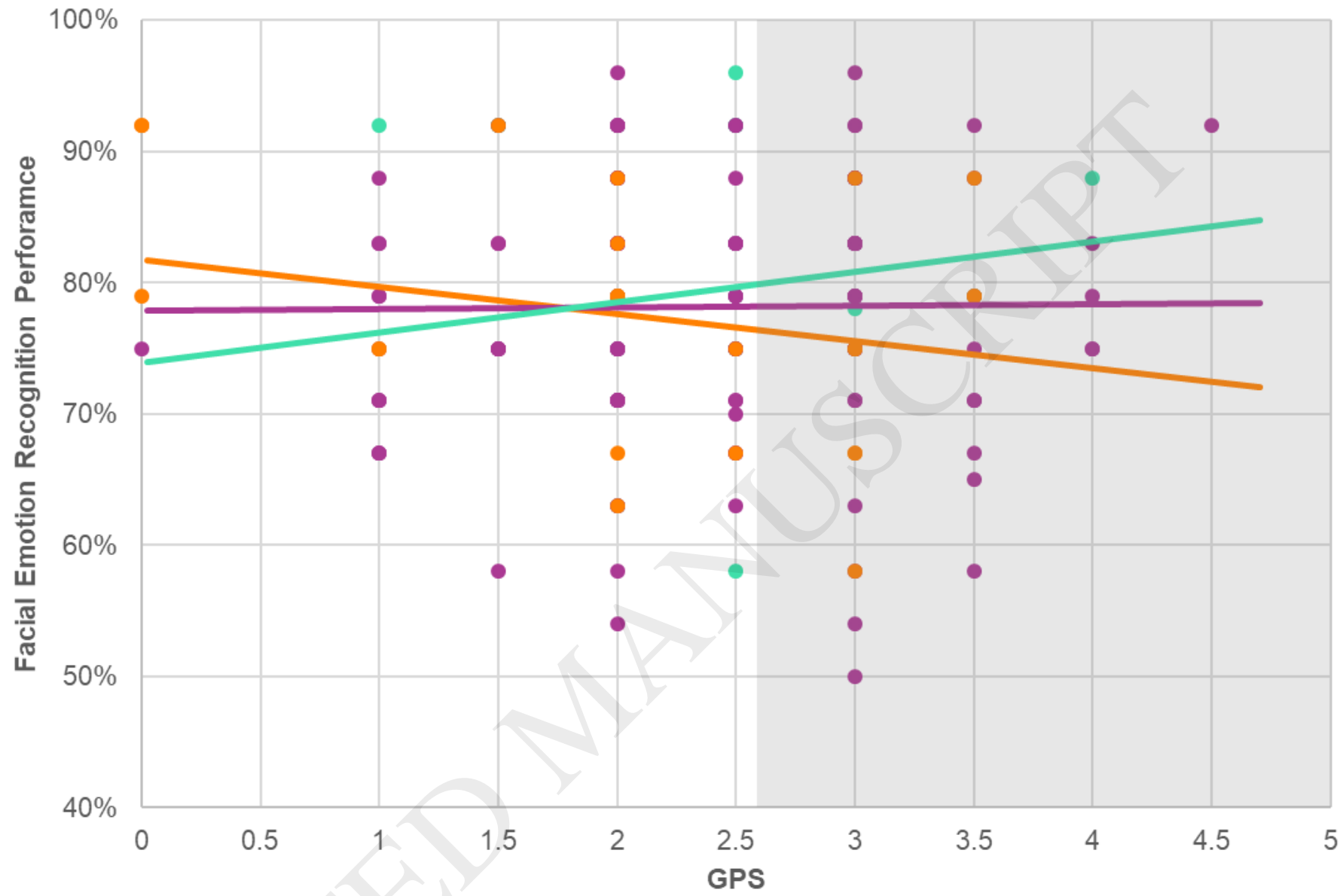

Figure 3 Facial emotion recognition plotted against genetic profile scores (raw data, grouped according to CTQ scores: CTQ <27 (M$\mathrm{SD}),<43$ and $>43(\mathrm{M}+\mathrm{SD})$ ). Simple slopes from post-hoc analyses at M-SD, M, and M+SD. J-N-analyses revealed a significance transition point at 2.55, i.e., for subjects with a GPS $>2.55$ there is a significant negative effect of CTQ on facial emotion recognition. 
Hartling et al: Interaction of HPA axis genetics and early life stress shapes emotion recognition in healthy adults

\begin{tabular}{|c|c|c|c|c|c|c|c|c|}
\hline Gene & SNP & Alleles & MAF & HWE $p$ & $\begin{array}{l}\text { Coded } \\
\text { variant }\end{array}$ & $\begin{array}{l}\text { Genetic } \\
\text { model }\end{array}$ & Coding rule & \\
\hline CRHRI & rs110402* & $\mathrm{G}>\mathrm{A}$ & .468 & .56 & A & recessive & $\mathrm{AA}=1 \quad \mathrm{GA}=0$ & $\mathrm{GG}=0$ \\
\hline CRHRI & rs4792887 & $\mathrm{C}>\mathrm{T}$ & .087 & .48 & $\mathrm{~T}$ & additive & $\mathrm{TT}=1 \quad \mathrm{CT}=0.5$ & $\mathrm{CC}=0$ \\
\hline$F K B P 5$ & rs1360780* & $\mathrm{C}>\mathrm{T}$ & .312 & .84 & $\mathrm{~T}$ & dominant & $\mathrm{TT}=1 \quad \mathrm{CT}=1$ & $\mathrm{CC}=0$ \\
\hline$F K B P 5$ & rs4713902 & $\mathrm{T}>\mathrm{C}$ & .282 & .18 & $\mathrm{C}$ & dominant & $\mathrm{CC}=1 \quad \mathrm{CT}=1$ & $\mathrm{TT}=0$ \\
\hline$N R 3 C l$ & rs41423247* & $\mathrm{G}>\mathrm{C}$ & .345 & .19 & G & recessive & $\mathrm{GG}=1 \quad \mathrm{CG}=1$ & $\mathrm{CC}=0$ \\
\hline $\mathrm{NR} 3 \mathrm{Cl}$ & rs6198 & $\mathrm{T}>\mathrm{C}$ & .152 & .57 & G & additive & $\mathrm{AG}=0.5$ & $\mathrm{AA}=0$ \\
\hline
\end{tabular}

Table 1 SNPs included in the genetic profile score, minor allele frequencies (MAF) and Hardy-Weinberg-equilibrium (HWE) and coding rules for contribution to the GPS. SNPs also included in Pagliaccio et al. 2014 are marked with asterisks. 
Hartling et al: Interaction of HPA axis genetics and early life stress shapes emotion recognition in healthy adults

\begin{tabular}{llcc|cc}
\hline & & \multicolumn{2}{c|}{ Step 1 } & \multicolumn{2}{c}{ Step 2 } \\
Step & & $\boldsymbol{\beta}$ & $\mathbf{p}$ & $\boldsymbol{\beta}$ & $\mathbf{p}$ \\
\hline $\mathbf{1}$ & Age & .180 & .312 & .118 & .472 \\
$\mathbf{1}$ & Gender & .243 & .165 & .258 & .109 \\
$\mathbf{1}$ & CTQ & .063 & .712 & .026 & .870 \\
\hline $\mathbf{2}$ & GPS & & & $\mathbf{. 4 2 0}$ & $\mathbf{. 0 0 8}$ \\
\hline & Model R & & 0.082 & & 0.251 \\
& Model Adjusted R $\mathbf{R}^{\mathbf{2}}$ & & 0.005 & & 0.166 \\
& Model F & 1.069 & & 2.938 \\
& Model p & & .374 & & $\mathbf{. 0 3 4}$ \\
& $\boldsymbol{\Delta} \mathbf{R}^{\mathbf{2}}$ & & & & $\mathbf{. 1 6 9}$ \\
& Change F & & & & $\mathbf{7 . 9 2 8}$ \\
& Change p & & & & $\mathbf{0 . 0 0 8}$ \\
\hline
\end{tabular}

Table 2 Regression analysis of cortisol increase on genetic profile score (GPS). Standardised beta- and p-values are presented for each predictor. For each step of the model, the $R^{2}$, adjusted $R^{2}, F$-, and $p$-value are presented, as well as the p-value for change in $R^{2}$ at each step. Any predictor, model or change in $\mathrm{R}^{2}$ significant at $\mathbf{p}<0.05$ is boldened. 
Hartling et al: Interaction of HPA axis genetics and early life stress shapes emotion recognition in healthy adults

Table 3 Regression analysis of facial emotion recognition on Childhood Trauma Questionnaire (CTQ), genetic profile score (GPS), and their interaction. Standardised beta- and p-values are presented for each predictor. For each step of the model, the $\mathbf{R}^{2}$, adjusted $\mathbf{R}^{2}$, Fand p-value are presented, as well as the p-value for change in $R^{2}$ at each step. Any predictor, model or change in $R^{2}$ significant at $\mathbf{p}<0.05$ is boldened.

\begin{tabular}{|c|c|c|c|c|c|c|c|}
\hline \multirow[b]{2}{*}{ Step } & & \multicolumn{2}{|c|}{ Step 1} & \multicolumn{2}{|c|}{ Step 2} & \multicolumn{2}{|c|}{ Step 3} \\
\hline & & $\boldsymbol{\beta}$ & $\mathbf{p}$ & $\boldsymbol{\beta}$ & $\mathbf{p}$ & $\boldsymbol{\beta}$ & $\mathbf{p}$ \\
\hline 1 & Age & -.464 & $<.001$ & -.446 & $<.001$ & -.438 & .000 \\
\hline 1 & Gender & -.204 & .003 & -.218 & .002 & -.238 & .001 \\
\hline 1 & IQ & .114 & .129 & .120 & .122 & .120 & .115 \\
\hline 2 & CTQ & & & -.095 & .181 & -.136 & .056 \\
\hline 2 & GPS & & & -.013 & .856 & -.003 & .971 \\
\hline 2 & Age $x$ GPS & & & -.008 & .915 & .018 & .808 \\
\hline 2 & Gender x GPS & & & .002 & .979 & .005 & .938 \\
\hline 2 & IQ x GPS & & & .064 & .387 & .074 & .305 \\
\hline & & & & .044 & .647 & .093 & .334 \\
\hline 2 & Age x CTQ & & & & & & \\
\hline 2 & Gender x CTQ & & & -.098 & .230 & -.109 & .175 \\
\hline 2 & IQ x CTQ & & & -.016 & .863 & -.021 & .814 \\
\hline 3 & CTQ x GPS & & 7 & & & -.190 & .009 \\
\hline & Model R ${ }^{2}$ & & .247 & & .265 & & .297 \\
\hline & Model Adjusted $\mathbf{R}^{2}$ & & .234 & & .214 & & .243 \\
\hline & Model F & & 18.162 & & 5.183 & & 5.525 \\
\hline & Model p & & $<.001$ & & $<.001$ & & $<.001$ \\
\hline & $\Delta \mathbf{R}^{2}$ & & .247 & & .018 & & .032 \\
\hline & $\Delta \mathbf{F}$ & & 18.162 & & .485 & & 7.093 \\
\hline & $\Delta p$ & & $<.001$ & & .866 & & .009 \\
\hline
\end{tabular}

